\title{
Laser Cleaning of a First Phase Navajo Wool Chief's Blanket by Nd:YAG
}

\author{
Pablo Londero ${ }^{1 \star}$, Ludovica Corda ${ }^{2}$, Daniele Ciofini $^{3}$, Christine Giuntini $^{4}$, \\ Marco Leona ${ }^{4}$
}

1 Institute for the Preservation of Cultural Heritage, Yale University, 300 Heffernan Rd., West Haven, CT 06516

2 Conservator in private practice, 315 King street, Apt. 5H, Port Chester, NY 10573

3 Institute of Applied Physics "Nello Carrara" IFAC, Via Madonna del Piano 10, 50019 Sesto Fiorentino, Florence, Italy

4 The Metropolitan Museum of Art, 1000 Fifth Avenue, New York, NY 10028

* Corresponding author: pablo.londero@yale.edu

\begin{abstract}
A case study was performed for laser cleaning of a soiled First Phase Navajo chief's blanket using the first and second harmonics of a Nd:YAG laser. A cleaning protocol was developed by mapping the impact of wavelength, fluence, pulse number, and surface pre-wetting on churro wool reference samples. Afterward, an approach for applying the protocol to the Navajo blanket was developed, including a number of custom jigs for mounting the object under controlled tension. The most effective laser cleaning parameters were determined to be a wavelength of $532 \mathrm{~nm}$, a fluence of $350 \mathrm{~mJ} / \mathrm{cm}^{2}$, approximately 100 pulses per cross-sectional area of the laser beam, and no pre-wetting. Good results with no observable damage were obtained with $\sim 280$ hours of laser cleaning for the entire blanket, which measured $138 \mathrm{~cm}$ by $208 \mathrm{~cm}$.
\end{abstract}

Keywords: laser cleaning, Navajo, wool, Nd:YAG

\section{Introduction}

Laser cleaning is increasingly used to remove unwanted contamination from the surfaces of historical materials, and has drawn significant interest for such applications on natural and man-made polymers [1-10]. Much of this work involves treatments on paper, such as the abatement of foxing, mold, and soiling [3, $5,6,8]$. Increased attention has been paid to the treatment of textiles woven from natural fibers, and a number of interesting results show the potential as well as limitations of laser cleaning, particularly for the removal of fine soiling and carbonaceous deposits [ 1 , $2,9,10]$.

Studies have shown that 1064 and 532 $\mathrm{nm}$ laser wavelengths, being less absorbed by most natural fibers, are more selective than UV irradiation in removing soiling. UV laser wavelengths can interact strongly with natural fibrous materials, either of vegetal or animal origin, thus resulting to be ineffective and potentially dangerous toward 
the structural integrity of fibers itself [2]. The side effects of this photo-damage are mainly described in terms of discoloration (i.e. yellowing or bleaching) and can be more easily controlled at longer wavelengths, where the interaction is lessened. At $1064 \mathrm{~nm}$ and $532 \mathrm{~nm}$, the laser light often interacts strongly with most inorganic environmental contaminants and weakly with the fiber itself, especially when the latter is not dyed or pigmented. The pollution particles tend to be broadband (black or brown) absorbers with thermal times scales on the order of hundreds of nanoseconds to microseconds [11], and are thus also well-suited to the use of Nd:YAG lasers.

As with many art conservation problems, a challenge for compiling useful data comes from the limitless variety of fabrics that can be created by combining different fibers, yarns and woven structures. A change in any one of these parameters can affect the propagation of laser light, ejected particulates, and any resulting damage to the fabric itself. Furthermore, since these materials involve many different polymers with various aging kinetics, the long-term impact of the treatment can also potentially vary. Additional thorough studies of well-selected fabrics and reference materials are needed in order to build toward the level of knowledge and intuition that already exists for the laser cleaning of materials such as stone and metal. Therefore, the following case study, which purpose-woven churro wool reference material in order to develop safe laser cleaning parameters for cleaning an historical churro wool Navajo blanket, provides a valuable example of laser cleaning on a class of materials of significant historical importance.

Wool harvested from churro sheep gradually became an important textile fiber throughout the American Southwest from the $17^{\text {th }}$ through the early $19^{\text {th }}$ centuries. Purposefully selected for their hardiness in the arid climate of New Spain by the Colonial authorities, the Iberian breed was imported into the Rio Grande valley in the late $16^{\text {th }}$ century. Their long staple fiber and clean fleece was ideally suited to the minimal hand-processing methods practiced by both the Spanish colonists and the Pueblo peoples. After the Pueblo revolt of 1680 , flocks of churro sheep were acquired by other Native American tribes including the Navajo [12].

This work focuses on a case study of laser cleaning of for a privately-owned First Phase Navajo Chief's Blanket mad of churro wool. Cleaning of artificially soiled (hereafter, "sooted") woven churro wool reference material by Nd:YAG was studied under varying parameters including wavelength, fluence, pulse number, and surface pre-wetting. Colorimetric and tensile properties were characterized before and after cleaning, with and without accelerated aging. The resulting data was used to develop a cleaning protocol for the Chief's Blanket. Once the treatment of the historical Navajo weaving was underway, additional techniques were developed as the protocol was adapted to the object.

\section{Materials and Methods}

\subsection{Case Study Description}

The historical object of concern is a privately owned First Phase Navajo Chief Blanket, which was brought to the Department of Scientific Research (DSR) at the Metropolitan Museum of Art (MMA) to investigate a particularly disturbing discoloration. The textile appeared to have been "home-cleaned" by a previous owner, and the original creamy white churro wool now exhibited a dingy 
mottled gray surface, punctuated by irregular splotches of bright white wool. According to the owner, a specialist restorer of traditional southwestern textiles tried to reduce the gray color, but the deeply ingrained soil was resistant to all the currently acceptable cleaning formulas used on this type of historic textile. Instrumental analysis indicated that the soiling was likely due to a carbonaceous deposit. This fact suggested that laser cleaning would be a possible treatment option if it could be demonstrated that the wool substrate would not be damaged.

Our tests focused on laser cleaning at 1064 and $532 \mathrm{~nm}$ with a Quanta System Thunder art High Power Q-switched Nd:YAG Laser for several reasons. The Metropolitan Museum owns such a laser system, which supports 1064, 532 and 355 $\mathrm{nm}$ outputs. However, the $355 \mathrm{~nm}$ output was not deemed to be worth testing as it would be too destructive to the wool substrate at the necessary fluences [2].

\subsection{Reference Sample Preparation}

In order to test the effect of the laser removal on carbonaceous deposits in churro wool, a custom-woven, weft-face churro wool fabric from sheep raised in the Southwest was selected as reference material. A portion of the woven fabric was cut into 12 pieces measuring approximately $15 \mathrm{~cm}$ by $15 \mathrm{~cm}$, and the edges were singed to prevent fraying during testing.

Afterward, laser cleaning experiments were planned according to two distinct application procedures of the artificial sooting material. The first of these involved samples that were sooted very evenly but not as deeply into the weave, the second one, samples that had fine carbon ink soaked into them. While this method placed the soot more unevenly, it also mimicked the Navajo blanket more accurately.

Evenly sooted samples were prepared using lab-made, water-based ink. A custom ink allowed us to apply an ink of pure soot, without the traditional stabilizers such as gum Arabic. $11.5 \mathrm{~mL}$ of DDI water and 125 mg of Kremer Furnace Black were combined in a stoppered glass vial. The vial was then placed in a sonication for four iterations 30 s each, with 10s of vigorous shaking after each sonication. Afterward, a pipet was inserted $\sim 2 / 3$ deep into the volume of ink, and 50\% of the contents were pipetted into a second clean glass vial. The second vial was sonicated and shaken twice as just described, and the contents were then pipetted into the inkwell of an airbrush for application. The ink was sprayed evenly until the reflectance was reduced by $50 \%$. Colorimetric measurements were performed across each sample to ensure $<3 \%$ variation in application. Four additional pieces of test material, $14 \mathrm{~cm}$ by $27 \mathrm{~cm}$, were prepared in the same fashion: the top and lower quarter of the textile were masked and the ink was applied only to the center portion (see Fig. 1a), so that cleaning tests at a given set of parameters could be performed side by side on unsoiled and sooted areas.

Deeply sooted samples were prepared using india ink (Fig. 1b). The ink was sprayed onto the reference wool, then pressed and smeared manually while wearing nitrile gloves. This process was repeated until a relatively even tone was achieved for the sooting throughout the reference samples.

\subsection{Laser Treatments}

Irradiation tests were performed using the fundamental radiation $(1064 \mathrm{~nm})$ and the second harmonic $(532 \mathrm{~nm})$ of a commercial Q-Switched (8 ns) Nd:YAG laser 
(ThunderArt, Quanta System S.p.A.). Laser cleaning performance was initially studied on evenly sooted samples (Fig. 1a). Laser fluence settings were selected to span the cleaning tests were performed on $2 \mathrm{~cm} \times 2$ $\mathrm{cm}$ test areas for every possible combination of selected parameters including wavelength (532 nm and $1064 \mathrm{~nm}$ ), fluence (532 nm, $5 \mathrm{ns:}$

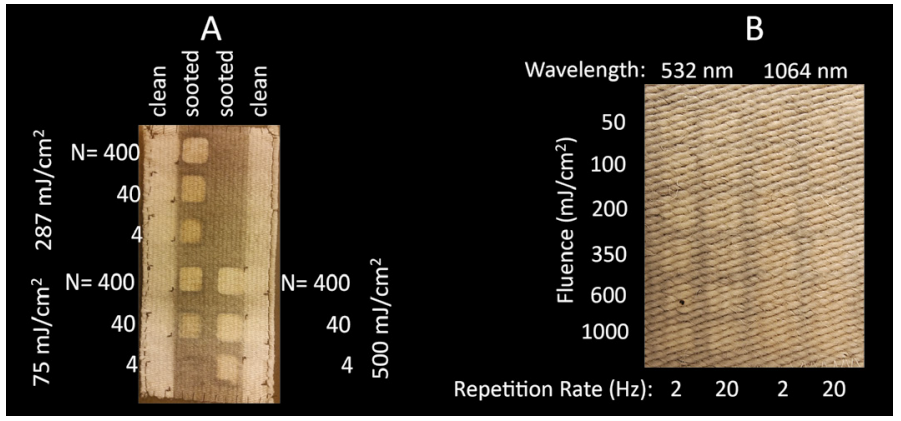

Fig. 1. Examples of reference samples for laser cleaning tests of heavily sooted (A) and deeply sooted (B) churro wool. range between cleaning threshold (judged visually after 10 pulses) and damage threshold, defined as $1 / 4$ of the fluence required to produce visible microscopic damage after 10 pulses. Pulse numbers were selected to explore laser cleaning behavior in both sudden and gradual cleaning regimes. This was deemed particularly important since gradual cleaning with many pulses was desired in order to controllably achieve visual uniformity across the blanket, but it was also important to minimize the risk of damage to the wool. Finally, surfaces were tested with pre-wetting and without, as both approaches have been shown to be beneficial in varying case studies.

In all laser cleaning experiments, laser pulse number was characterized in terms on pulses per laser beam area. This was due to the need to vary the beam diameter in order to obtain some of the greater fluences. The number of pulses per treated area thus varied, but the number of pulses per beam area was a unit of measure proportional to the flux applied over a treated region. A programmable, motorized stage was used to control the number of pulses per laser spot area. Laser
60, 360, $660 \mathrm{~mJ} / \mathrm{cm}^{2} ; 1064 \mathrm{~nm}, 6 \mathrm{~ns}: 75,287$, $\left.500 \mathrm{~mJ} / \mathrm{cm}^{2}\right)$, pulses per beam area $(4,40$, and 400), wool surface (unsoiled and sooted), and pretreatment just before cleaning (pre-wetting and no pre-wetting). A repetition rate of $4 \mathrm{~Hz}$ was selected to achieve reasonable cleaning rates while avoiding generating damage from gradual, continuous heating. This value was arrived at empirically from initial testing in which thermal damage was observed at repetition rates above $10 \mathrm{~Hz}$ and fluences of $350 \mathrm{~mJ} / \mathrm{cm}^{2}$ or higher, which was attributed to heat transfer from contaminants embedded within the purchased reference material.

Based on the results of the study above, additional experiments were planned on deeply sooted samples (Fig. 2a) in order to explore the most viable cleaning parameters under conditions that were less repeatable but more representative of a genuine treatment by a conservator. Instead of a motorized stage, in this experiment, samples were irradiated by scanning manually the laser beam over $1 \times 1 \mathrm{~cm}^{2}$ of surface using a fixed working distance of $12.5 \mathrm{~cm}$ from the target and a spot diameter of about $5 \mathrm{~mm}$ at both the wavelengths. 
Cleaning performance as a function of fluence was systematically tested from $50 \mathrm{~mJ} /$ $\mathrm{cm}^{2}$ up to $1 \mathrm{~J} / \mathrm{cm}^{2}$ for both the wavelengths and the repetition rate was 2 (exp. $50 \mathrm{sec}$ ) and 20 (exp. $5 \mathrm{sec}$ ) Hz. In this way an equal number of laser pulses $(\mathrm{N}=100)$ per spot was delivered. Further tests aimed at assessing the laser-induced color changes on untreated (no sooting to clean) wool were carried out using 350, 600 and $1000 \mathrm{~mJ} / \mathrm{cm}^{2}$ and 2 (exp: $50 \mathrm{sec}$ ), 10 (exp: $10 \mathrm{sec}$ ) and 20 (exp: $5 \mathrm{sec}$ ) $\mathrm{Hz}$, as shown in Fig. 5.

\subsection{Analytical Techniques}

Color measurements were performed using a custom setup consisting of Ocean Optics HL-2000-HP tungsten-halogen source fiber-coupled to an integrating sphere with a 6 mm opening, with the spheres optical output fiber-coupled to an Ocean Optics USB 2000+ spectrometer. Data were collected with a spectral resolution of $2 \mathrm{~nm}$ in the 380-780 $\mathrm{nm}$ range. Calibration was performed by means of $99 \%$ diffuse reflectance standard and CIE $\mathrm{L}^{*} \mathrm{a}^{*} \mathrm{~b}^{*}$ color coordinates were calculated considering $2^{\circ}$ degrees standards observer values. The behavior of test areas in CIE- $L^{*} a b$ color space was characterized in terms of deviations from the original, unsoiled color. In order to properly interpret the colorimetric data, 16 measurements of clean, original textile areas were made in different sections of the reference material, while four colorimetric measurements were taken in laser treated areas. The color variation between the laser cleaned surfaces and the average values for the original surface are expressed as $\Delta \mathrm{L}, \Delta \mathrm{a}$ and $\Delta \mathrm{b}$ and $\Delta \mathrm{E}$ (CIE Lab 2000 color definition).

A final set of accelerated light aging experiments were also prepared, to test the long-term optical response of the wool to the most promising laser cleaning parameters. Cleaned areas of interest were placed in an Atlas ci35 fadeometer at $100 \mathrm{Klx}$ for two weeks, simulating 37.5 years of 50 lux exposure for 10 hours a day. Colorimetric measurements were taken after exposure to compare with pre-existing measurements, using the same setup described above. Accelerated thermal was performed at 60 Celsius and $75 \% \mathrm{RH}$ for three weeks in a Caron 7000-33-1 environmental chamber, approximating 60 years of aging at 20 Celsius and $65 \% \mathrm{RH}$. Longitudinal tensile response for individual fibers was recorded both before and after accelerated aging in accordance with the ASTM D3822-14 protocol, using an Instron 4443 Universal Testing System. Tensile performance of individual fibers was characterized by the offset yield strength, defined as the stress at which the stress-strain curve for axial loading deviates by a strain of $1 \%$ from the linear-elastic line.

Samples for tensile testing were created by performing laser cleaning on both clean and sooted reference sample areas. Sooting was performed in accordance with the protocol for evenly sooted samples, described above. Cleaning of four $2 \mathrm{~cm} \times 2 \mathrm{~cm}$ areas was performed at $350 \mathrm{~mJ} / \mathrm{cm}^{2}$ with 400 pulses per beam area, on both sooted and original areas. After accelerated thermal aging, fifteen fibers were selected from each location. Only those fibers that were most exposed at the surface of the yarns were chosen, as they experienced the greatest amount of sooting and laser radiation and/or therefore the most extreme laser cleaning environment. All fibers were inspected by microscope under transmitted light at 400X magnification for any noticeable laser-induced damage. Fiber lengths of 15.7 $\mathrm{mm}$ were then mounted across plexiglass tabs under mild pretension (as specified in the ASTM standard) using two-component 
epoxy. They were then allowed to dry and acclimated in the same climate-controlled room where the testing was performed, at 23 Celsius and 50\% RH. After testing, the fiber were weighed on a Mettler Toledo Excellence Plus microbalance in order to properly scale the tensile data. The sum of the experiments allowed us to obtain a window of settings for adequately cleaning the blanket without significant damage.

\section{Results and Discussion}

\subsection{Reference Material Study}

In order to properly interpret the colorimetric data, 16 measurements of clean, original textile areas were made in different sections of the reference material. A natural variation of $1.57,0.17,0.8$, and 1.62 was found for $\Delta L$, $\mathrm{D} a, \mathrm{D} b$, and $\Delta \mathrm{E}$, respectively.

The colorimetric results from laser cleaning tests on evenly sooted samples are shown in Figs. 2 and 3. The behavior of test areas in CIE- $L^{\star} a b$ color space was characterized in terms of deviations from the original, unsoiled color (Fig. 2). Changes in value for $\mathrm{L}, a, b$, and the resulting $\Delta \mathrm{E}$ (CIE Lab 2000 definition), between the cleaned surfaces and the average values for the original surface are shown. The measurements reveal a number of clear, instructive patterns. Two broad, general trends are the improved performance of dry samples over pre-wetted ones, and the poor performance of cleaning at $1064 \mathrm{~nm}$ in comparison to the $532 \mathrm{~nm}$ wavelength.

The deterioration of cleaning performance on a wetted surface, as measured by the $\Delta \mathrm{L}$ coordinate is evident at both wavelengths. Cleaning of a dry surface outperforms that of a wet surface for all but the highest fluence and pulse number settings. Performance differences in the $\Delta L$ coordinate between "dry" and "wet" are comparable only at fluences of approximately $0.5 \mathrm{~mJ} / \mathrm{cm}^{2}$ and 400 pulses per beam area. Overall improvement in cleaning at $532 \mathrm{~nm}$ over $1064 \mathrm{~nm}$ is similarly evident. Lightening from soot removal, as characterized by the $\mathrm{L}$ coordinate, requires a high fluence and 400 pulses to achieve the same contrast reduction that the $532 \mathrm{~nm}$ treatment attains at with 40 pulses at nearly one tenth of the fluence.

Other smaller, but still clear, patterns in behavior show that treatments with both $532 \mathrm{~nm}$ and $1064 \mathrm{~nm}$ wavelengths have the capability to induce color change beyond that caused by soot removal. As $532 \mathrm{~nm}$ pulses are applied at greater fluence and number, the wool both shifts to higher values of $L$ and more negative values of $a$, corresponding to a slight lightening and greening in hue. The lightening is evident only at the highest fluence and maximum pulse number of 400 , while the change in hue is perceptible for both medium and high fluence at 400 pulses. It is unlikely that the change is caused by the removal of the added soot, since similar changes are present in clean areas with the same treatment applied. While this color change may partially be due to the removal of additional dirt that is present in the original reference material, it is unlikely to be the cause of the change in hue. This suggests a slight alteration of the material dependent on the pulse peak energy and total pulse number, potentially due either to accumulated brief heating from individual pulses or to previously reported photo-responsive changes in wool's reflective properties that are depend on the illuminating wavelength [13].

For the case of $1064 \mathrm{~nm}$, a certain amount of darkening remains with respect to the original color, even at high fluence and 400 pulses. Additionally, significant and consist- 

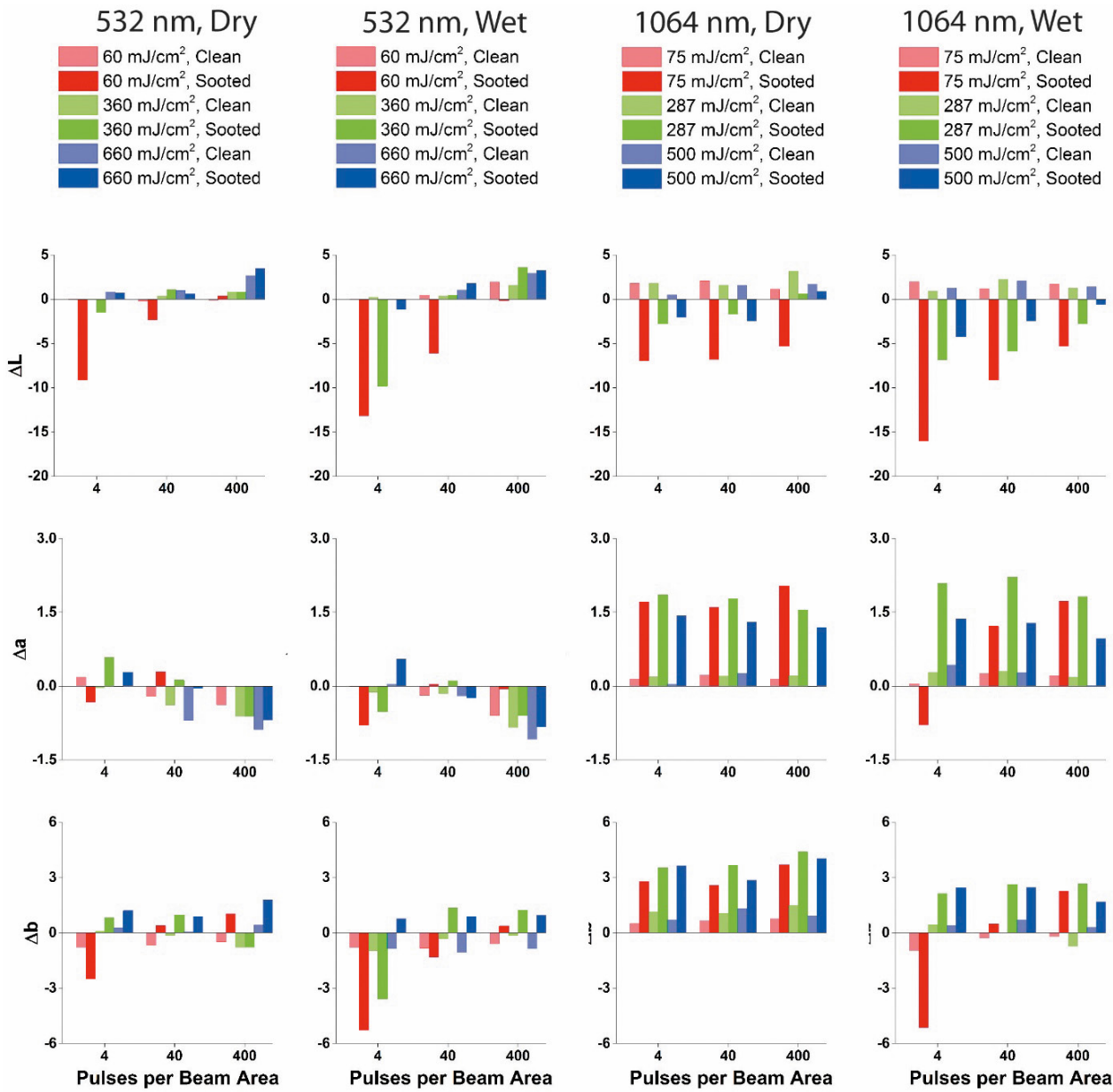

Fig. 2. $\Delta L, \Delta a$, and $\Delta b$ values of colorimetric data for laser cleaning tests of heavily sooted and nearby unsoiled areas. The difference between the laser cleaned area and a nearby original (unsoiled, uncleaned) area is plotted. Wavelength, surface pre-treatment, fluence, and pulse number are varied.

ently positive shifts in coordinates $a$ and $b$ exist at all power levels and pulse number, but only in the areas that were sooted. This last point suggests that the color change, which corresponds to reddening and is clearly visible, is most likely due to remnants or byproducts of the cleaned soot that were not effectively removed by the infrared laser. This was informally supported by cleaning an additional area at medium fluence with
$1064 \mathrm{~nm}$ to generate the red stain, and then removing the red stain by passing over the same area at comparable fluence with $532 \mathrm{~nm}$ pulses. Similar behavior has been observed for removal of carbonaceous deposits on gypsum by $1064 \mathrm{~nm} \mathrm{Nd:YAG} \mathrm{pulses} \mathrm{[14,15].}$ These experiments showed that nanoparticles of remnant soot-derived material are present in colored areas, and that the presence of other organic compounds can enhance the 
development of colored deposits. However, the remaining deposits remain sensitive to and can be removed by shorter wavelengths. It is possible that the sooted wool samples experience similar behavior: $1064 \mathrm{~nm}$ wavelengths generate photochemistry of organic impurities to produce colored substances that do not interact strongly with the ablating laser, or the ablation dynamics create soot-derived nanoparticles during that are not sufficiently absorbing.

Overall, the colorimetric results from the data above suggest that the most interesting range of parameters for laser cleaning of sootlike deposits from churro wool are fluences at or below $360 \mathrm{~mJ} / \mathrm{cm}^{2}$, with tens to hundreds of laser pulses per pulse area, at $532 \mathrm{~nm}$ and without pre-wetting the surface. The analysis
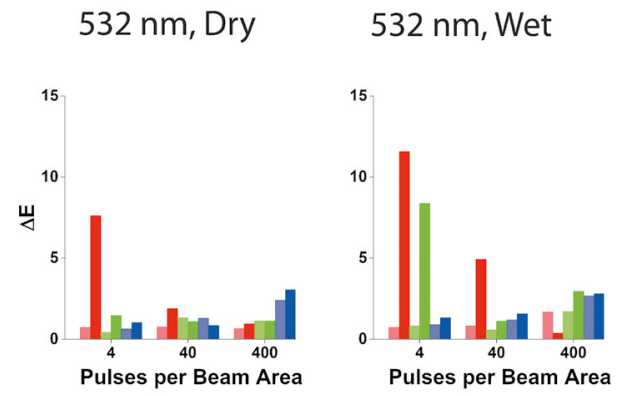

pressed india ink more precisely clarifies the optimal power, pulse number, and repetition rate for laser cleaning deposits on churro wool. As expected, irradiation at $532 \mathrm{~nm}$ generates marked increases of $\Delta L$ in comparison with the $1064 \mathrm{~nm}$ wavelength. In contrast, $\Delta a$ rises as function of laser fluence in major extent at $1064 \mathrm{~nm}$, thus confirming what was previously observed in cleaning tests of heavily sooted samples (Fig. 2). The removal process at $1064 \mathrm{~nm}$ is drastically less efficient than at $532 \mathrm{~nm}$ and the yellow-red color remaining on the surface at $1064 \mathrm{~nm}$ is most likely due to residual ink particles that are not sufficiently absorbing at $1064 \mathrm{~nm}$, as observed with the previously described, more heavily sooted samples. Instead, the 532 $\mathrm{nm}$ laser wavelength produces, just starting

$$
1064 \text { nm, Dry } 1064 \text { nm, Wet }
$$
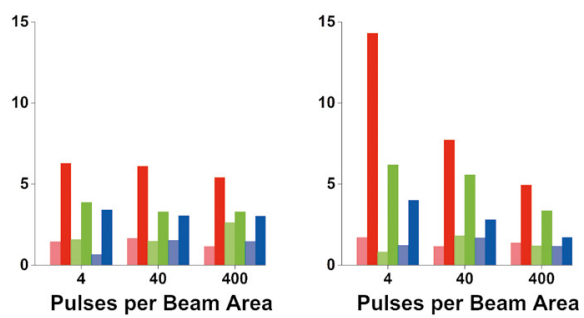

Fig. 3. $\Delta \mathrm{E}$ values for laser cleaning tests of heavily sooted samples, corresponding to the data shown in Fig. 2.

of overall color change from the above measurements $(\Delta \mathrm{E})$, shown in Fig. 3, shows how the general behavior of the laser cleaning experiments supports this conclusion. A consistent color which is within measurement error of the original is obtained throughout this parameter range. We note that previous studies of laser-cleaned animal fibers by SEM have shown morphological change can occur at a threshold that is approximately half that of any visible change in color [2].

The analysis of more focused experiments performed on the samples with soaked and from the lower fluences tested, a high degree of particle removal and it may be therefore considered most suited for reaching appreciable cleaning results on sooted wool textiles. $\Delta b$ increases with increasing fluences as a consequence of soot removal but at the same time, its behavior is similar at both the wavelengths. It is worth noting that the color variations observed in Fig. 4 all have a saturation trend with increasing fluence, and in most cases, become constant around $150-200 \mathrm{~mJ} / \mathrm{cm}^{2}$ at $532 \mathrm{~nm}$ and among $200-350 \mathrm{~mJ} / \mathrm{cm}^{2}$ at $1064 \mathrm{~nm}$. No significant 

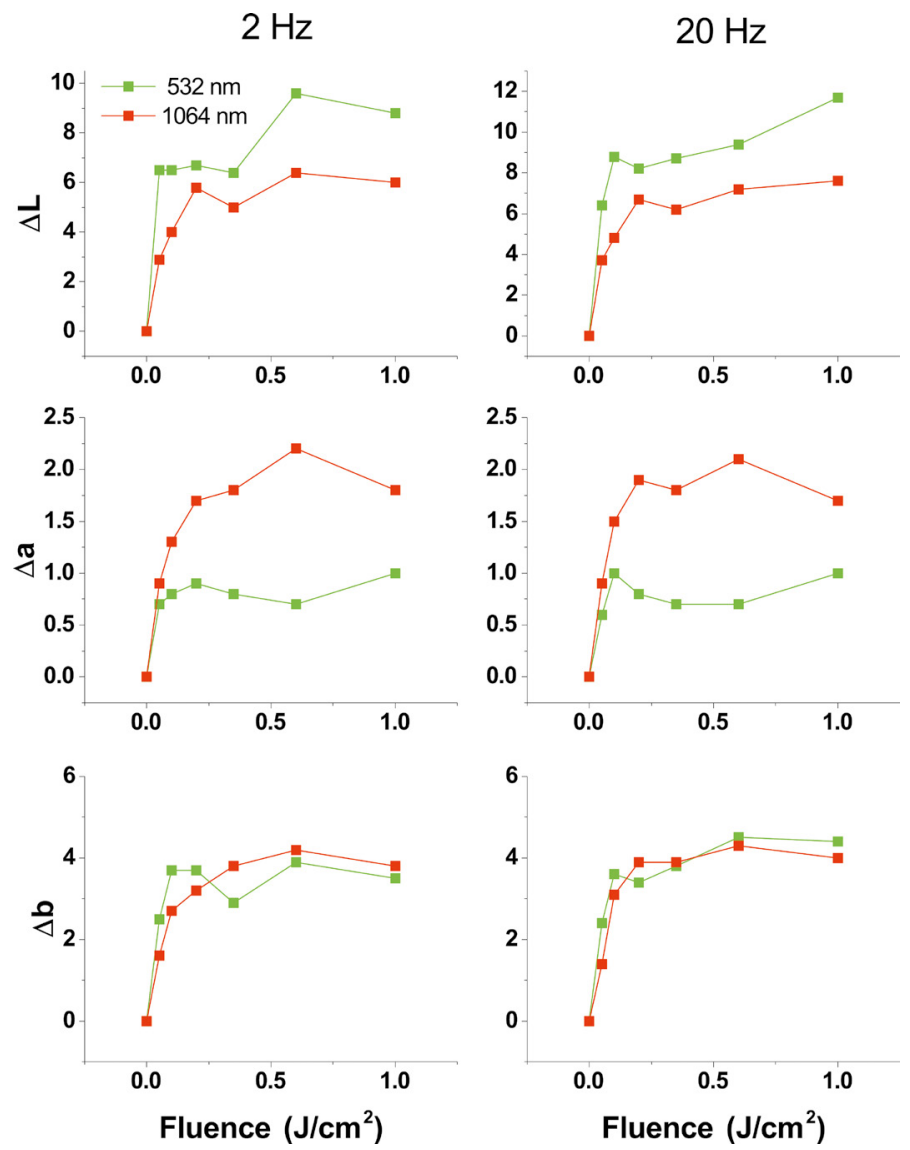

Fig. 4. $\Delta L, \Delta a$, and $\Delta b$ values, with respect to the sooted surface before cleaning, for deeply sooted samples. Using 100 pulses per beam area repetition rate and fluence are varied. Note that improvements in cleaning generally saturate beyond $\sim 350 \mathrm{~mJ} / \mathrm{cm}^{2}$.

differences were observed between $2 \mathrm{~Hz}$ and $20 \mathrm{~Hz}$ operation.

As expected, all the above-mentioned considerations are reflected in the $\Delta \mathrm{E}$ variation, where the slight color difference between the two wavelengths is further demonstrated (Fig. 5).

Data exploring the margin of safety for the optimal parameters above, by varying repetition rate and relatively high fluence rates, is shown in Fig. 6 and Fig. 7. Firstly, $\Delta L, \Delta a$ and $\Delta b$ undergo variation with respect to the original surface that tends to increase more so with the number of laser pulses delivered and less with the fluence and repetition rate. However, variations of
$\Delta L$ appear to be negligible up to $600 \mathrm{~mJ} / \mathrm{cm}^{2}$, either at 2, 10 and $20 \mathrm{~Hz}$.

The opposite trend may be clearly observed in the irradiation tests carried out at $1 \mathrm{~J} / \mathrm{cm}^{2}$. In fact, $\Delta \mathrm{L}$ increases as function of laser pulses and repetition rates, until reaching a constant value of about 3 at 20 $\mathrm{Hz}$. This change is sufficient to be visible to the naked eye, and the irradiated area seems to be brighter. In contrast, $\Delta$ a decreases with increasing laser pulses, beginning at approximately 60 laser pulses per beam diameter. Although the $\Delta \mathrm{b}$ change is quite negligible, it tends to increase, thus suggesting the color change proceeds toward a yellow or green color hue, consistent with the other reference 

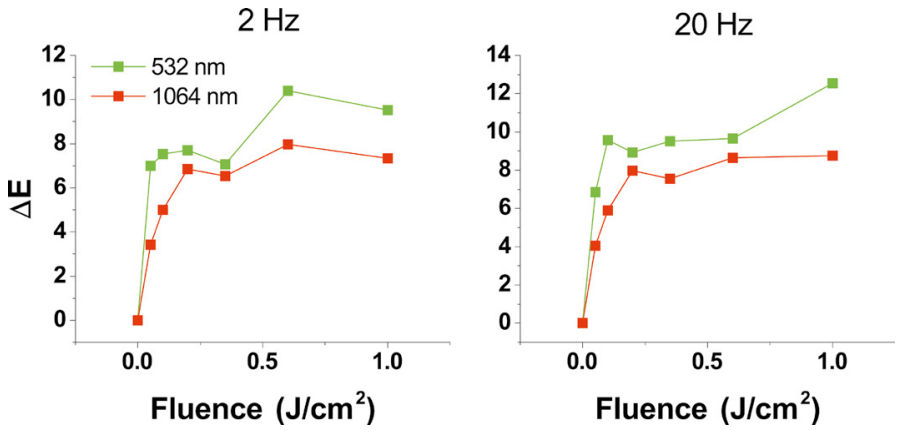

Fig. 5. $\Delta \mathrm{E}$ values for laser cleaning tests of deeply sooted samples, corresponding to the data shown in Fig. 4.


$\begin{array}{lllll}20 & 40 & 60 & 80 & 100\end{array}$

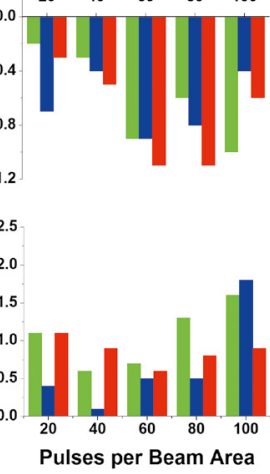

Fig. 6. $\Delta L, \Delta a$, and $\Delta b$ values of colorimetric data for laser cleaning tests of unsoiled samples at $532 \mathrm{~nm}$ with no pre-wetting. The difference between the laser cleaned area and a nearby original (unsoiled, uncleaned) area is plotted. Fluence, pulse number, and repetition rate are varied.

sample results. The variations in $\mathrm{D} a$ and $\mathrm{D} b$ variations are small; however, they present a clear direction for color change as a result of prolonged exposure to laser irradiation.

Trends for $\Delta \mathrm{E}$ under the various conditions are shown in Fig. 7. These are in most cases lower than the material's natural variation of $\Delta \mathrm{E}=1.6$. However, at fluences of $1 \mathrm{~J} / \mathrm{cm}^{2}$ and $20 \mathrm{~Hz}$ color change is the most prominent $(\Delta \mathrm{E} \geq 3)$, independently from the number of pulses. In this case, the occurrence of laser-induced color changes takes place at certain energy density and repetition rate, possibly due to localized cumulative heating. In fact, previous laser irradiation tests aimed at finding macroscopic damage thresholds on single-spot basis, showed that at $350 \mathrm{~mJ} / \mathrm{cm}^{2}$ and $20 \mathrm{~Hz}$ no surface changes were observed after 30 seconds of exposure. Contrarily, a dark brown spot, likely due to cumulative heating, was clearly observable at $600 \mathrm{~mJ} / \mathrm{cm}^{2}, 20 \mathrm{~Hz}$ after 20 seconds and at 1 

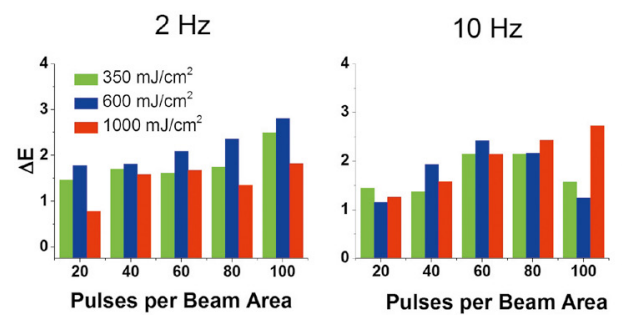

$\mathrm{J} / \mathrm{cm}^{2}, 20 \mathrm{~Hz}$ after 10 seconds. The measured damage thresholds for the untreated wool sample under study are therefore dependent from the total radiant exposure $\left(\mathrm{J} / \mathrm{cm}^{2} \cdot \mathrm{Hz}\right)$ integrated over time.

It is therefore worth noting while some color change is primarily a function of the total number of pulses, which suggests a damage mechanism dependent on the peak energy, other effects take place at very high fluence and repetition rates, which suggests a thermal effect due to constant heating. This second effect, however, is far from the optimal cleaning conditions $\left(350 \mathrm{~mJ} / \mathrm{cm}^{2}\right.$, $10-20 \mathrm{~Hz}$ ). The wool appears to be thermally and mechanically stable to laser irradiation below $600 \mathrm{~mJ} / \mathrm{cm}^{2}$. However, due to the low thermal diffusivity of organic material (for wool thermal conductivity $k$ is $0.03 \mathrm{~W} /(\mathrm{m} \cdot \mathrm{K})$ at $25^{\circ} \mathrm{C}, \rho=1.3 \mathrm{~g} / \mathrm{cm}^{3}$ and $C_{p}=1.357 \mathrm{~J} / \mathrm{g} \cdot \mathrm{K}$, and the calculated thermal diffusivity $D=1.7$ $\cdot 10^{-4} \mathrm{~cm}^{2} / \mathrm{s}$ ) [16-18], it is advisable to move the beam rapidly over the surface to avoid any undesired effect. In addition, thermal damage could be drastically increased if there is prolonged exposure for large absorbing impurities (dirt and pollutants) that remain trapped between fibers, or for melanin-rich or colored fibers.

As described in the section 2.4, accelerated light and thermal aging tests were used to probe for any long-term effects due to laser cleaning. Results from the former are shown in Fig. 8. While the treated areas

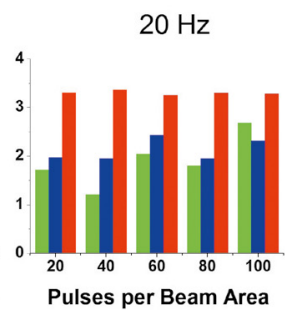

Fig. 7. $\Delta \mathrm{E}$ values for laser cleaning tests of deeply sooted samples, corresponding to the data shown in Fig. 6.

demonstrated an overall color change in comparison to the untreated area that was slightly greater than the one standard deviation of measurement error, the results show no consistent pattern. The unsoiled area cleaned with 40 pulses per beam area showed a greater difference than that cleaned with 400 pulses, while the sooted area cleaned with 40 pulses per beam area showed less difference than that cleaned with 400 pulses. We therefore concluded that the effects were most likely due to slightly greater variation of the initial material in this area, and not the treatment itself. In all of the above cases,

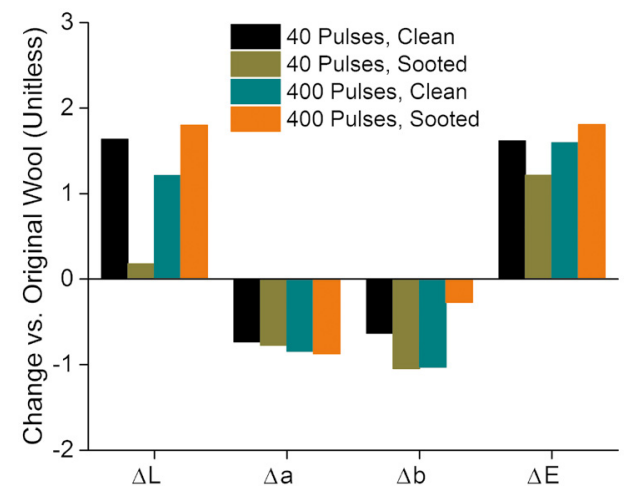

Fig. 8. $\Delta L, \Delta a, \Delta b$, and $\Delta \mathrm{E}$ values for of colorimetric data for laser cleaning tests of the heavily sooted sample laser cleaned at $532 \mathrm{~nm}$ and $350 \mathrm{~mJ} / \mathrm{cm}^{2}$ with no pre-wetting, for pulse numbers of 40 and 400 on both sooted and original (clean) surface areas. The difference between the laser cleaned area and a nearby original (unsoiled, uncleaned) area is plotted. Pulse number is varied. 


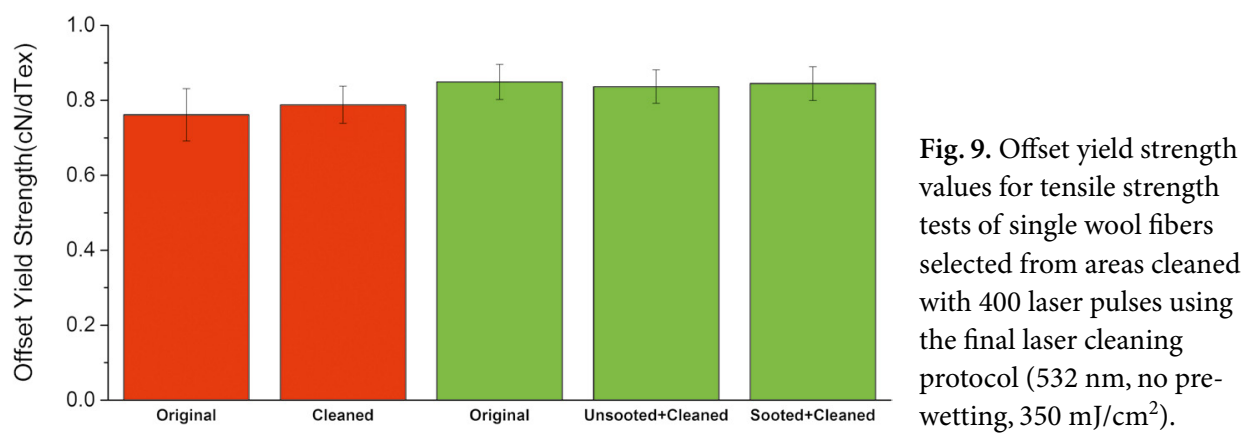

no damage was visible under microscopic examination.

Tensile measurements after accelerated thermal aging are shown in Fig. 9. The offset yield strength does not change appreciably between the original fibers and those that have been sooted and cleaned. This is the case both with and without thermal aging. It is possible that a slight trend of generally increased offset yield strength after thermal aging (red vs. green) is emerging, although more statistics would be needed to verify this. This would likely be due to a slight embrittlement after thermal aging, which is expected behavior for wool fibers.

The cumulative data from both studies shows that optimal laser cleaning of a churro wool blanket should occur at $532 \mathrm{~nm}$ and approximately $350 \mathrm{~mJ} / \mathrm{cm}^{2}$, on a dry surface with up to 100 pulses per beam area. These settings should clean soot-like deposits efficiently without any noticeably color change, whether by eye or using colorimetric instrumentation. One should also expect to see no visible change in the individual fibers, even under 400X magnification.

\subsection{Case Study, Laser Cleaning of a First Phase Navajo blanket}

The physical condition of the Chief Blanket, shown in Fig. 10, was assessed. It was examined by eye at several magnifications, ranging from $5 x$ to $100 x$. The yarn make-up and details of the woven structure were recorded. Although the aesthetic appearance of the blanket was poor, the physical condition was very good. The wool fibers remain strong as do the yarns produced from them. Overall, the woven structure was intact; several internal damages had been expertly rewoven. The selvage twining had also been restored and was intact. The corner tassels were also intact and appear to be in original condition. Importantly, when the blanket was viewed through transmitted light, it was possible to document the small areas where the weft was thinned as a result of abrasion, likely from use and prior cleanings.

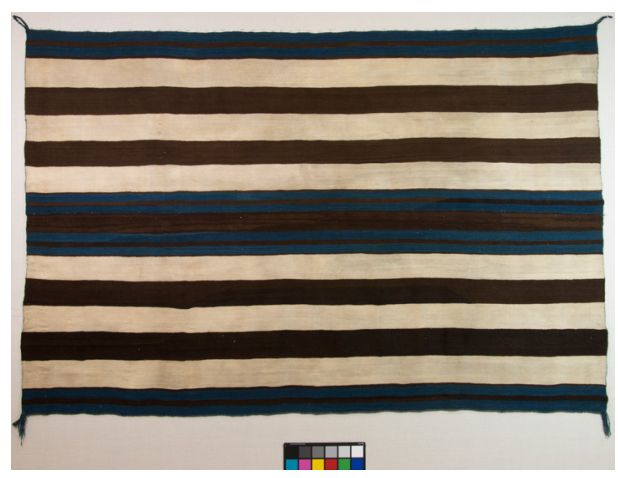

Fig. 10. Image of the "Back" surface of the Navajo blanket. The blanket measures $54-1 / 2$ " $(138 \mathrm{~cm})$ by $82 "(208 \mathrm{~cm})$. Fabrics are elastic objects. These are the maximum dimensions, excluding tassels. 
The major condition issue was the uneven coloration of the undyed white churro wool: it was unevenly stained a sooty gray color on both faces, and these areas were punctuated by a variety of white "stains" where all the gray color had been removed. An example of these conditions is shown in Fig. 11 (left). It was presumed that the uneven gray deposits were also distributed over the blue and brown bands, but were not visible, leaving their appearance unaffected. The likely composition of soiling was determined by reflectance spectroscopy. This method was chosen for several reasons. To begin with, sampling was not permitted. Since the soiling was extremely fine and dilute, and since very little material is needed to produce noticeable color variation, non-invasive vibrational spectroscopy techniques such as Raman spectroscopy and FTIR were only sensitive to the wool. Microscopic examination was also inconclusive: it was unclear whether the particles located were in fact the main contributors to the color variation.

Typical reflectance data is shown in Fig. 11 (right). The contaminant was characterized by measuring the ratio of reflectance from a clean area to that from a soiled area. The resulting spectrum is consistent with sooting: a relatively flat response over a very broad range of wavelengths, with a gradual decrease in reflectance toward the near-infrared [19].

As described in the previous section, the tests run on the hand-woven churro reference fabric indicated that soot removal was more efficient at $532 \mathrm{~nm}$ pulses than at $1064 \mathrm{~nm}$ pulses. During the first hours spent getting familiar with the Nd:YAG laser, the conservators practiced on the churro reference sample and on a large damaged fragment of a Navajo weaving, specifically purchased by the owner for this purpose. Combinations of frequencies $(5,10$, and $20 \mathrm{~Hz})$ and beam size at the surface were tested and the results were compared, in an effort to determine which parameters would deliver a more consistent and effective cleaning. In order to maintain the fluence of the laser beam below $350 \mathrm{~mJ} / \mathrm{cm}^{2}$ and avoid damage to the substrate, the beam diameter at the surface was kept at or above $5-7 \mathrm{~mm}$. The optimum treatment parameter was a setting of $532 \mathrm{~nm}$ at $20 \mathrm{~Hz}$ with a beam size at the surface of approximately $6 \mathrm{~mm}$. This setting ensured the soot particles would be removed evenly (i.e. uniformity of color) within a reasonable amount of time.
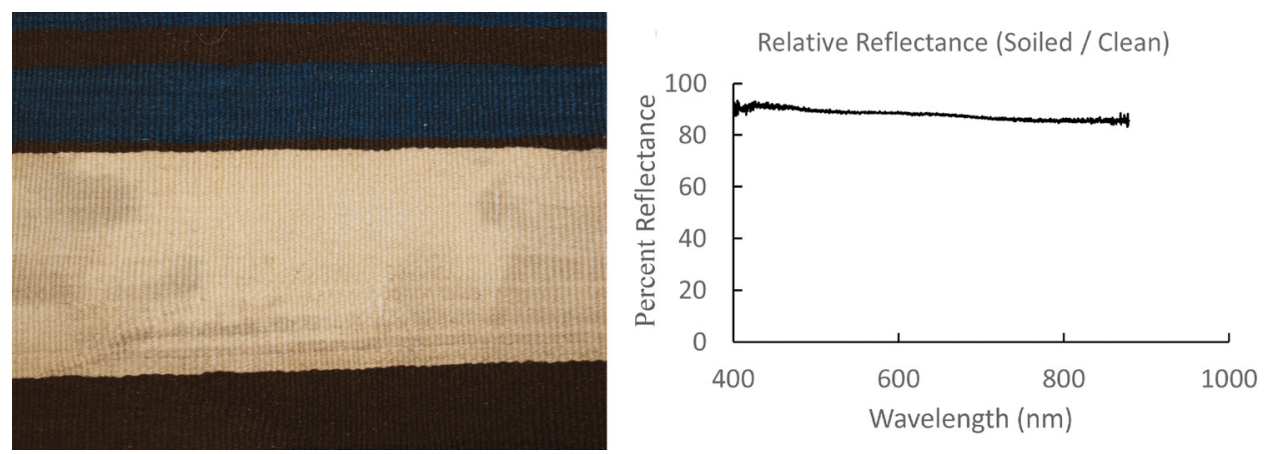

Fig. 11. (Left) Example of the various effects of the soiling, including large areas of discoloring that perhaps indicate a previous cleaning test or attempt, as well as finer variations in darkening throughout. (Right) Typical spectrum for the percent difference in reflectance between soiled and unsoiled areas of the blanket. 
In order to systematically clean the white bands, a label was added to one face of the weaving. The cleaning was begun at the bottom right band. The area to be treated was marked by pins at the left and right sides. Initially, photographs were taken for comparative purposes, but this time-consuming process was discontinued when it became apparent that the protocol was effective. The blue and brown yarns were masked with aluminum foil, pinned into place. The pins were secured into a $1 / 2$ " thick sheet of Ethafoam ${ }^{\circ} 990$ covered with aluminum foil. The aluminum foil would reflect the beam, rather than possibly allowing heat to be absorbed by the underlying foam (see Fig. 12 A). Empirically, it was observed that a small amount of tensioning in the warp direction facilitated the cleaning process. It is presumed that tensioning allows a greater surface area of weft was exposed to the laser beam. It was also determined empirically that tensioning in the weft direction would also facilitate cleaning.

Approximately half of the cleaning was accomplished using the Ethafoam ${ }^{\circ}$ sheet. However, it became clear that some of the soiling remained resistant to the laser, especially in areas near the bright stains. Since the tests with the churro samples revealed that the undyed wool substrate was not affected by the laser at the recommended parameters, and in consultation with the owner, a second pass of the laser was carried out. For this second cleaning, a curved jig was created that would allow more surface area of the wool to be exposed. It was constructed from a section of $31 / 2$ " archival tube, cut in half along the length and secured to a sheet of the Ethafoam ${ }^{\circ}$ 990. This curved form was then covered with aluminum foil. This tool reduced the surface area that could be warp-tensioned during a cleaning session, thus increasing the manipulation of the cloth. However, the curved surface allowed the laser to further penetrate the weft interstices for a more in depth cleaning. This tool, originally used to treat areas that had been already cleaned on the flat foam surface, soon replaced it altogether, when it became apparent that even with more frequent moving and repinning, the cleaning time was reduced. Its use is shown in Fig. 12 B.

There remained areas that retained an eye-catching uneven coloration. The owner approved the testing of a third tool, assembled from 4-inch Styrofoam ${ }^{\text {tw }}$ hemisphere mounted on a cylindrical Ethafoam ${ }^{\circ} 660$ base. It was developed so that small areas of both the warp and weft could be simultaneously tensioned. This tool, called "the dome" (see Fig. 12 C) significantly reduced the area
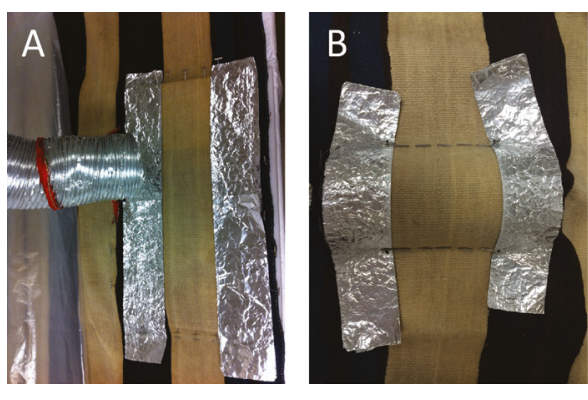



Fig. 12. View of table from top: (A) A section of the blanket prepared for cleaning by stretching horizontally over an Ethafoam ${ }^{\circ}$ sheet; (B) A section of the blanket prepared for cleaning by tensioning horizontally over a half-tube jig;

(C) The three jigs used during laser cleaning of the Navajo blanket. 
that could be cleaned, but allowed the conservators to focus on very specific areas. Again, the evenness of the cleaning was assessed and a fourth and final tool, dubbed "the saddle" (see Fig. 12 C), and made of a tall block of Ethafoam $^{\circ} 660$ that was carved into a shape resembling a narrow horse saddle, was used to focus the cleaning on specific weft interstices and transition lines resulting from the use of the half-tube during the previous cleaning phase. Only about four passes of the weft were exposed during the cleaning and the laser was manipulated to clean around the "crown" of each weft. This final support jig was the most complicated to tension, but it did allow the conservators to remove enough of the resistant soot to even out more of the undyed wool surface. Although it may have been possible to remove more soot, the amount of time under the laser was not equal to the quality of the results, and the cleaning was ended. Afterward, treatment photographs of both faces of the Chief Blanket were taken, along with selected details.

Although it was not possible to do before and after tensile testing of this identically faced tapestry woven Chief Blanket, no damage to the wool was observed at $100 \mathrm{X}$ magnification. Samples were not extracted for higher level magnifications. For both the conservators, scientists and the owner, the look and the handle of the weaving was unchanged. The brown and indigo wools were covered during the treatment and remain unchanged.

Results of the cleaning are shown in Fig. 13. Overall lightening from soot removal is clearly evident when the blanket is folded over to contrast a cleaned area with one that is yet uncleaned (see Fig. 13 A). Additionally, while the white bands remain unevenly colored, the contrast in particularly irregular areas such as that shown in Fig. 11 (left) has been significantly reduced (see Fig. 13 B, C). The eye-catching white stains are not immediately evident, although traces remain, but only to those trained to look for them. The areas where the weft was thin and fragile were unchanged and no losses in these areas occurred during the treatment. Perhaps natural aging will reveal some now-hidden agent of deterioration, but given the amount of pre-treatment testing on churro wool, this event seems unlikely.
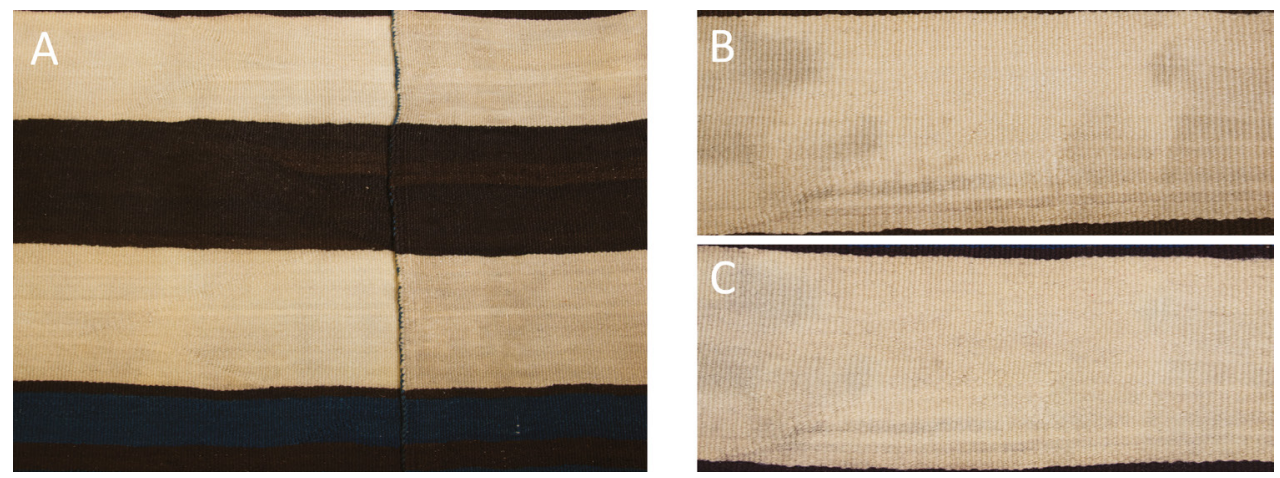

Fig. 13. (A) A section of the blanket is folded over show the difference between cleaned (lighter) and uncleaned (darker) areas of the blanket; (B) A region of the uncleaned blanket with particularly large variation in lightness; (C) The region shown in $12 \mathrm{~B}$, after laser cleaning. 


\section{Conclusion}

We have successfully performed laser cleaning of sooty soiling on a wool First Phase Navajo chief blanket, based on laser parameters that were optimized through an extensive study of woven churro wool reference material. The reference study showed an optimal balance between cleaning effectiveness and negligible damage at settings of $532 \mathrm{~nm}$ and $350 \mathrm{~mJ} / \mathrm{cm}^{2}$, with no pre-wetting of the wool surface. These parameters left no discernable damage even with hundreds of pulses applied per beam area, and accelerated light and thermal aging tests did not exhibit a difference between original and laser-cleaned surfaces. Cleaning of the Navajo blanket resulted in significant overall removal of soiling, exhibited by lightening of the surface color, and reduction of contrast between clean and previously soiled areas. The wool fibers of the cleaned blanket were found to undamaged based on macroscopic, microscopic, and tactile examination of the blanket following treatment.

\section{Acknowledgements}

We gratefully acknowledge Quanta System for providing the Thunder Art Laser to the Metropolitan Museum of Art and Dr. Edgar O. Smith for his support. We would also like to acknowledge Robert Mann and Robert Mann Rugs for providing the reference material.

\section{References}

[1] C. Escudero, M. Martinez, P. Moreno, and A. Arranz, "Study of laser cleaning of ancient fabric with femtosecond pulses", in: Lasers in the conservation of artworks: proceedings of the International Conference LACONA VII, Madrid, Spain, September 17-21, 2007, M. Castillejo, P. Moreno, M. Oujja, R. Radvan,
J. Ruiz (Eds.), CRC Press, Inc., Boca Raton, Florida, 2013, pp. 337-343.

[2] R. Belli, A. Miotello, P. Mosaner, and L. Toniutti, "Laser cleaning of artificially aged textiles", Applied Physics A, 83 (2006) 651-655, DOI: 10.1007/s00339006-3530-3.

[3] D. Ciofini, I. Osticioli, S. Micheli, L. Montalbano, and S. Siano, "Laser removal of mold and foxing stains from paper artifacts: preliminary investigation", Proc. SPIE 9065, (2013), 906512-1 - 906512-11, DOI: $10.1117 / 12.2052820$.

[4] C. Rosati, D. Ciofini, I. Osticioli, R. Giorgi, S. Tegli, and S. Siano, "Laser removal of mold growth from paper," Applied Physics A, 117 (2014) 253-259, DOI: $10.1007 /$ s00339-014-8507-z.

[5] T. Ersoy, T. Tunay, M. Uğuryol, G. Mavili, and S. Akturk, "Femtosecond laser cleaning of historical paper with sizing", Journal of Cultural Heritage, 15 (2014) 258-265, DOI: 10.1016/j.culher.2013.07.002.

[6] S. Pentzien, A. Conradi, and J. Krüger, “The influence of paper type and state of degradation on laser cleaning of artificially soiled paper", in: Lasers in the conservation of artworks: proceedings of the International Conference LACONA VIII, Sibiu, Romania, September 21-25, 2011, R. Radvan, J. F. Asmus, M. Castillejo, P. Pouli, A. Nevin, (Eds.), CRC Press/ Balkema, Leiden, Netherlands, 2011, pp. 59-65.

[7] T. Fourrier, G. Schrems, T. Mühlberger, J. Heitz, N. Arnold, D. Bäuerle, M. Mosbacher, J. Boneberg, and P. Leiderer, "Laser cleaning of polymer surfaces", Applied Physics A, 72, (2001) 1-6, DOI: 10.1007/ s003390000683.

[8] W. Kautek, S. Pentzien, D. Mueller-Hess, K. Troschke, R. Teule, "Probing the limits of paper and parchment laser cleaning by multi-spectral imaging", Proc. SPIE 4402 (2001) 130-138, DOI: 10.1117/12.445655.

[9] H. Sutcliffea, M. Cooperb, J. Farnswortha, "An initial investigation into the cleaning of new and naturally aged cotton textiles using laser radiation", Journal of Cultural Heritage, 1 (2000) S241-S246, DOI: 10.1016/S1296-2074(00)00178-3.

[10] A. Elnaggar, P. Fitzsimons, A. Nevin, K. Watkins, M. Strlic, "Viability of laser cleaning of papyrus: conservation and scientific assessment", Studies in 
Conservation, 60 (2015) S73-S81, DOI: 10.1179/003 9363015Z.000000000211.

[11] F. Liu, M. Yang, F. Hill, D. Snelling, G. Smallwood, "Influence of polydisperse distributions of both primary particle and aggregate size on soot temperature in low-fluence LII", Applied Physics B, 83 (2006) 383-395, DOI: 10.1007/s00340-006-2196-Z.

[12] J. Wheat, Blanket Weaving in the Southwest, University of Arizona Press, Tucson, 2003.

[13] H. Launer, "Effect of Light Upon Wool: Part VI: Spectral Conformity in Photobleaching of Wool", Textile Research Journal, 36 (1966) 606-611, DOI: $10.1177 / 004051756603600703$.

[14] C. de Oliveira, P. Bromblet, A. Colombini, V. Vergès-Belmin, "Medium-wave ultraviolet radiation to eliminate laser-induced yellowing generated by the laser removal of lamp black on gypsum", Studies in Conservation, 60 (2015) S34-S40, DOI: 10.1179/0039363015Z.000000000205.
[15] C. de Oliveira, V. Vergès-Belmin, D. Demaille, P. Bromblet, "Lamp black and hematite contribution to laser yellowing: A study on technical gypsum samples", Studies in Conservation, 61 (2016) 136-145, DOI: $10.1179 / 2047058415 Y .0000000003$.

[16] Z. Ye, C. Wells, C. Carrington, N. Hewitt, “Thermal conductivity of wool and wool-hemp insulation", International Journal of Energy Research, 30 (2006) 37-49, DOI: 10.1002/er.1123.

[17] The Engineering ToolBox, http://www.engineeringtoolbox.com/specific-heat-capacity-d_391. html, (accessed 01.10.2016).

[18] Aqua-Calc, http://www.aqua-calc.com/page/ density-table/substance/wool, (accessed 01.10.2016).

[19] R. Levinson, P. Berdahl, H. Akbari, "Solar spectral optical properties of pigments-Part I: model for deriving scattering and absorption coefficients from transmittance and reflectance measurements", Solar Energy Materials and Solar Cells, 89 (2005) 319-349, DOI: 10.1016/j.solmat.2004.11.012. 
